\title{
Explorando o Impacto da Gamificação na Redução do Gaming the System em um Ambiente Virtual de Aprendizagem
}

\author{
Laís Zagatti Pedro, Seiji Isotani \\ Instituto de Ciências Matemáticas e de Computação (ICMC) \\ Universidade de São Paulo (USP) \\ laiszagatti@gmail.com, sisotani@icmc.usp.br
}

\begin{abstract}
Resumo. Um dos grandes problemas encontrados nos ambientes virtuais de aprendizagem atuais é o surgimento de comportamentos inadequados por parte dos alunos que acabam por reduzir os beneficios que estes ambientes oferecem. Dessa forma, esse trabalho tem por objetivo propor e validar o uso de técnicas de gamificação em um ambiente chamado E-Game para reduzir a externalização de comportamentos indesejáveis, em particular o gaming the system, comportamento típico de alunos que tentam trapacear para completar atividades de ensino associado a falta de interesse e motivação. Para avaliar a proposta foram realizados experimentos controlados com 70 alunos do ensino fundamental durante três semanas no contexto do ensino de matemática. Os resultados empíricos obtidos indicam que há forte correlação entre o uso da gamificação e a diminuição do gaming the system. Verificou-se também diferenças de comportamento e impacto motivacional entre alunos de gêneros diferentes. Alunos (gênero masculino) tiveram menos comportamentos indesejáveis durante o uso do ambiente gamificado enquanto que alunas (gênero feminino) se sentiram menos competentes ao usar o ambiente gamificado. Este é o primeiro trabalho que reporta a descoberta da relação entre elementos de gamificação, gaming the system e gênero, baseados em dados empíricos em ambiente real de aprendizagem abrindo espaço para novas pesquisas em uma área até então pouco explorada.
\end{abstract}

\section{Introdução}

Diversos estudos apontam que alunos que utilizam ambientes virtuais de aprendizagem frequentemente aprendem mais, melhorando o seu desempenho e retenção do conhecimento, se comparado com alunos de uma sala de aula convencional (Isotani et al., 2009; Simpson, 2013; VanLehn et al., 2014). Entretanto, apesar dos benefícios educacionais que esses ambientes proporcionam, ainda existem alguns problemas críticos que comprometem uma boa aprendizagem. O tédio, desinteresse, monotonia, a falta de motivação, entre outros fatores, acabam por fazer com que o aluno se comporte inadequadamente ao interagir com o ambiente educacional (Baker et al., 2008). Dentre os comportamentos inadequados mais estudados a trapaça no sistema, do Inglês "gaming the system", tem recebido destaque (Cetintas et al., 2010). Este comportamento é caracterizado quando o aluno tenta trapacear o sistema resolvendo os exercícios mecanicamente, sem levar em consideração o que precisa ser aprendido.

Avanços importantes para detecção desse tipo de comportamento foram alcançados pela comunidade (Muldner et al., 2011). Contudo, são poucas as pesquisas empíricas que estudam meios para amenizar este problema comportamental por meio do desenvolvendo e implementação de técnicas que aumentem a motivação dos alunos e, por consequência, contribuam para prevenir ou reduzir a externalização de comportamentos considerados 
prejudiciais a aprendizagem (Pedro et al., 2015). Mais raros ainda são estudos empíricos que verificam como essas técnicas afetam diferentemente alunos do gênero feminino e masculino.

Nesse contexto, esse trabalho tem por objetivo propor, implementar e avaliar uma alternativa para reduzir o comportamento indesejável, gaming the system, em ambientes educacionais Web por meio do uso de técnicas de Gamificação. Essas técnicas permitem aumentar a motivação e o engajamento do aluno, além de guia-lo para interagir de forma correta com o ambiente (Kapp, 2012; Borges et al., 2015). Para atingir o objetivo desejado, foram estudados os principais elementos de gamificação para sua posterior implementação em ambiente educacional. Também estudou-se como parametrizar o gaming the system para coletar dados específicos sobre este tipo de comportamento. E, por fim, desenvolveuse um ambiente educacional gamificado, chamado E-Game, para verificar como os elementos de jogos podem auxiliar na diminuição de comportamentos como o gaming the system, aumentando a motivação dos alunos durante o processo de ensino-aprendizagem.

Foram realizados um estudo piloto e dois experimentos controlados com alunos do ensino fundamental durante o aprendizado de matemática. Neste trabalho, será relatado o segundo experimento que teve a participação de 70 alunos do ensino fundamental. A partir das análises estatísticas comparativas entre um grupo de controle (ambiente sem gamificação) e um grupo experimental (ambiente gamifcado) realizadas apenas com alunos que completaram todas as tarefas $(\mathrm{N}=60)$ observou-se que há uma diferença significativa no comportamento dos alunos. Empiricamente, os resultados indicam que há uma diminuição do comportamento de gaming the system no grupo experimental que utilizou o ambiente gamificado E-Game. Além disso, observou-se uma diferença nos resultados relacionado ao gênero dos alunos, até então pouco reportada pela literatura de gamificação e educação (Dicheva et al., 2015; Klock et al., 2015). Foi detectado que o gênero masculino externalizou menos trapaças durante o uso do ambiente educacional gamificado, em relação ao gênero feminino. Por outro lado, o gênero feminino externalizou menos trapaças no ambiente não gamificado, em comparação ao ambiente gamificado.

Para descrever as atividades desenvolvidas neste trabalho, nas próximas seções estaremos apresentando os trabalhos relacionados, os procedimentos e métodos utilizados, o design dos experimentos, a análise dos dados obtidos e, por fim, serão discutidos os resultados alcançados e as conclusões desta pesquisa.

\section{Trabalhos relacionados}

Um mapeamento sistemático foi conduzida para tentar identificar trabalhos que utilizam a jogos ou gamificação para evitar ou reduzir comportamentos inadequados dos alunos, em particular, o gaming the system; e também identificar quais elementos de jogos mais contribuem para redução deste tipo de comportamento. A resultado completo deste mapeamento está disponível em Pedro (2016).

Diversos estudos constatam que a forma como o ambiente educacional é construído pode provocar uma maior tendência aos alunos adquirirem comportamentos indesejáveis como o gaming the system. Por exemplo, os resultados de Baker et al. (2009) sugerem que o comportamento de gaming the system é mais frequente em conteúdos e atividades que são abstratas, ambíguas, e que não possuem uma descrição clara. Além disso, o trabalho de Doddannara et al. (2013) apresenta evidências de que há relações significantes entre o estado emocional negativo do aluno e o surgimento de comportamentos inadequados. Assim, a inclusão de elementos de gamificação em ambientes de aprendizagem pode 
fornecer subsídios motivacionais aos alunos e, por consequência, reduzir o gaming the system.

Neste contexto, diversos estudos comparam o uso de ambientes de aprendizagem baseados em jogos e os ambientes tradicionais e, por meio de análises estatísticas, mostram que como é possível aumentar a motivação e comportamentos desejados. Um exemplo de estudo foi descrito por Hess e Gunter (2013), que comparam as experiências de aprendizado de cursos online baseado e não baseado em jogos. Os resultados mostram que ambientes baseados em jogos aumentam a motivação, a colaboração social, e produz uma melhora na atenção. Em outro estudo, Giannakos et al. (2012) mostraram a implementação do elemento de jogo storytelling em um ambiente educacional e identificaram que alunos que costumavam ter um desempenho baixo mostraram melhoras significativas de aprendizagem e concentração. Outro artigo interessante é o de Mayer at al. (2013) que compara ambientes de aprendizagem virtuais baseado em jogos procurando identificar qual é a vantagem ou possível desvantagem nas suas utilizações. Neste trabalho verificou-se uma melhora na atitude dos alunos e a correção com a satisfação do aluno durante a aprendizagem.

Apesar de existirem vários estudos sobre o assunto, a maioria dos artigos analisados no mapeamento utilizam jogos sérios para auxiliar na aprendizagem e não elementos de jogos em contextos de aprendizagem. Dessa forma, há evidências de uma lacuna de pesquisa na condução de estudos empíricos sobre a gamificação em ambientes virtuais de aprendizagem com foco na redução de comportamentos inadequados dos alunos. Este trabalho se insere nesta lacuna realizando estudos para entender como os elementos de jogos afetam os alunos e podem auxiliar na redução do comportamento gaming the system.

\section{E-Game: Plataforma para Estudo da Gamificação na Educação}

O E-Game foi proposto como um ambiente virtual de aprendizagem Web, desenvolvido na linguagem Java, utilizando o framework Model-View-Controller (MVC) open source VRaptor $^{1}$ com integração ao Hibernate ${ }^{2}$, usando banco de dados MySQL. A interface e design do E-Game utilizou o padrão "flat design" que preza uma simplicidade, clareza e honestidade de materiais em interfaces de usuário.

\subsection{Definição dos elementos de jogos no E-Game}

Após estudos juntamente com especialistas, foram selecionados elementos de jogos específicos para serem implementados no ambiente educacional a ser utilizado durante o experimento (PEDRO et al., 2015). Os elementos de jogos, combinados com os padrões de mecânica de jogos, são:

- PONTOS: Cada usuário acumula seus próprios pontos. Para cada questão correta, o usuário ganha 5 pontos.

- RANKING: O ranking funciona através dos pontos ganhos pelos usuários. O ranking pode ser personalizado, mostrando o avatar escolhido por cada usuário, e quantos pontos eles possuem.

- BADGES: O usuário recebe medalhas/insígnias diferentes de acordo com o número de perguntas que ele/ela responde corretamente.

\footnotetext{
${ }^{1}$ Framework de desenvolvimento para aplicações web, disponível em <http://www.vraptor.org/pt/>

${ }^{2}$ Framework de integração com banco de dados disponível em <http://hibernate.org/>
} 
- FEEDBACK: Em cada questão, o ambiente apresenta dois botões para o usuário. Antes de continuar para a próxima questão, o jogador deve verificar se a resposta está correta. Dessa forma, o ambiente mostra o feedback imediato para o usuário, mostrando se a resposta está correta ou não. Caso negativo, o jogador possui mais 2 chances para acertar cada questão. Além disso, um botão de Dicas está disponível a qualquer momento, e o ambiente mostra uma janela com 5 dicas para ajudar o jogador a responder a questão. Até a dica número 3, o jogador pode visualizar sem custo, a partir da dica 4, é necessária a compra a dica com seus pontos.

- INDICADORES: os indicares mostram estatísticas sobre o desempenho do aluno durante a interação com o ambiente como: quantidade de perguntas (certa/errada), quantidade de dicas solicitadas, quantidades de vidas perdidas (o aluno tem 3 tentativas para responder uma questão corretamente), tempo gasto nas atividades, etc.

Essa combinação de elementos de jogos e mecânicas foi proposta com o objetivo de evitar ou reduzir a externalização de comportamentos indesejáveis como Gaming the System através do aumento da motivação nos alunos mantendo o engajamento durante as atividades. A Figura 1 apresenta uma das telas do E-Game para resolução de exercícios múltipla escolha. No menu lateral existe uma maior concentração dos elementos de jogos implementados. De cima para baixo, primeiramente os pontos ficam em destaque no topo do menu, seguido das barras de progresso logo abaixo. A primeira barra (no formato redondo) é a quantidade de respostas corretas em porcentagem, ou seja, contando do total de questões que ainda está disponível e, logo em seguida, a barra de contagem das respostas erradas. Por fim na aba de progressos, a terceira barra é a geral do ambiente, que vai preenchendo conforme o usuário responde as questões. Abaixo das barras de progresso, os badges ganhos pelo usuário são exibidos em forma de troféu, a fim de incentivar mais o usuário a continuar acertando. E, por fim, a tabela de ranking é exibida com os primeiros 5 colocados da turma que está realizando a mesma atividade, de acordo com a pontuação de cada um, exibindo o avatar que cada usuário escolheu. Além do menu na barra lateral, a parte superior da tela exibe os indicadores que são números sobre a quantidade de perguntas respondidas corretamente, as erradas e quantas vezes o usuário solicitou a ajuda do ambiente. Ao final das questões respondidas, a tela do perfil mostra um resumo da interação do usuário, seu progresso, todos os seus badges e seu lugar no ranking na atividade desenvolvida.

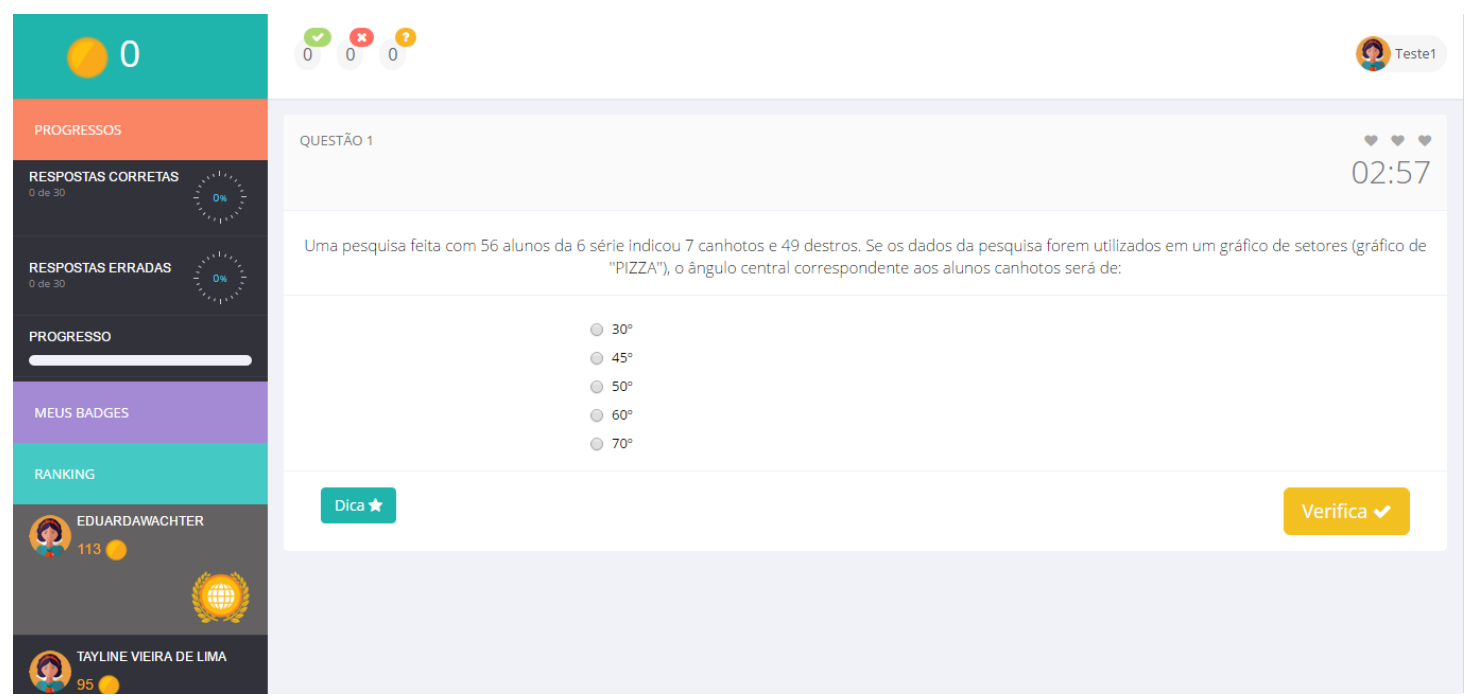

Figura 1. Tela de um problema de múltipla escolha no E-Game 
V Congresso Brasileiro de Informática na Educação (CBIE 2016)

Anais dos Workshops do V Congresso Brasileiro de Informática na Educação (CBIE 2016)

\subsection{Parametrização do Gaming the System}

A partir do ambiente educacional desenvolvido, foi necessário definir os parâmetros que serão analisados para identificar o comportamento indesejável do gaming the system. Esses parâmetros têm por objetivo mensurar se o aluno externalizou o comportamento indesejável. Os comportamentos considerados como gaming the system descritos por Baker et al. (2008) são: (i) Repetidamente e rapidamente solicitar por dicas (ajuda) durante a atividade; (ii) Tentativa sequencial de respostas rápidas e sistemáticas (do popular, 'chute' das respostas).

Há diversas formas de mensurar e registrar se está ocorrendo os tipos de comportamentos descritos por Baker et al. (2008). O comportamento $i$ é observado quando o aluno descobre que o ambiente educacional sugere dicas e/ou ajuda para a realização das questões e solicita essa ajuda durante a atividade repetidamente e rapidamente. Esse comportamento indica que ele não está interessado em ler a questão e em pensar na resolução do problema, tendo apenas o objetivo de conseguir a resposta correta. No contexto dos ambientes educacionais desenvolvidos, o arquivo de $\log$ registrou diferentes variáveis para serem analisadas e assim é possível detectar esse tipo de comportamento:

- Tempo na questão até a solicitação da dica: indica o tempo em que o sujeito permaneceu na página para ler a questão até o momento em que ele fez a primeira solicitação da dica e/ou ajuda.

- Tempo durante a dica: indica o tempo que o sujeito permaneceu com a dica e/ou ajuda aberta, demonstrando se ele leu o que está sendo sugerido ou não;

- Tempo entre as dicas: tempo de permanência em uma dica. Se um sujeito solicitar rapidamente múltiplas dicas, temos o indicativo que ele não as leu adequadamente.

- Quantidade de dicas solicitadas: através de uma análise estatística, essa variável demonstra o quão frequente a solicitação de dicas ocorreu.

O comportamento ii também sugere a análise do tempo verificando tentativas sequenciais, rápidas e repetidas na tentativa de acertar uma questão. No vocabulário popular nas escolas de hoje, esse comportamento é conhecido como 'chute' da questão, na qual o aluno não lê propriamente a questão e tenta acertar a alternativa correta, ou quando ele não sabe a resolução e clica repetidas vezes em alternativas aleatórias. Para análise e registro desse comportamento, foram propostas as seguintes variáveis:

- Tempo para responder: a análise do tempo desde que o aluno abre a questão até o momento em que ele escolhe uma alternativa.

- Quantidade de mudança de escolha: registra alterações na resposta do sujeito, independentemente se essa resposta é a resposta final submetida.

- Tempo entre a mudança da escolha: além da quantidade, é registrado o tempo entre as mudanças de respostas.

- Tempo para próxima questão: a análise do tempo de permanência na questão, até o momento em que o sujeito solicita uma próxima questão.

\section{Avaliação Experimental}

Durante todo o período do desta pesquisa, foram realizados um estudo piloto e dois experimentos. Devido a limitação de espaço, neste trabalho será relatado apenas o segundo experimento, que foi realizado com um número maior de estudantes. A principal questão de pesquisa a ser respondida neste trabalho foi: 
V Congresso Brasileiro de Informática na Educação (CBIE 2016)

Anais dos Workshops do V Congresso Brasileiro de Informática na Educação (CBIE 2016)

- QP1 - A gamificação aplicada em um ambiente educacional diminui a externalização do comportamento indesejado gaming the system durante uma atividade educacional? Existe diferença entre gêneros?

Para responder essa questão, realizou-se um experimento durante aproximadamente 3 semanas com 70 alunos do ensino fundamental, 60\% meninas $(\mathrm{N}=42)$ e $40 \%$ meninos $(\mathrm{N}=28)$, de uma escola estadual na cidade de São Carlos e foi dividido em 2 partes:

1. Pré-teste: como uma prova digital. É a aplicação de 30 questões elaboradas para identificar qual o nível de conhecimento dos alunos em matemática;

2. Intervenção: Nesta fase, metade dos alunos utilizaram o ambiente E-Game apresentado, com elementos de jogos (grupo experimental), e a outra metade utilizou o mesmo ambiente, mas sem os elementos de jogos (grupo de controle) que chamamos de E-Class. Durante a intervenção os alunos realizaram as seguintes atividades: Questionário pessoal: o aluno insere as suas informações como forma de cadastro no ambiente como nome, idade, gênero, série e data de nascimento. Exercícios: são apresentadas todos os conteúdos, funcionalidades, características e regras do ambiente. E em seguida o aluno responde a 30 questões de matemática de mesmo nível do pré-teste. Questionário motivacional: utilizou-se o IMI - Intrinsic Motivation Inventory (Deci \& Ryan, 2011) para aferir o estado emocional dos sujeitos composto por 22 questões que analisam a motivação intrínseca em 4 categorias: Interesse/Aproveitamento, Competência, Escolha e Pressão/Tensão.

\section{Resultados}

A análise principal foi realizada utilizando o seguinte cenário comparativo: E-Game $x$ EClass (i.e. grupo experimental vs. grupo controle); e observando as variáveis relatadas na seção 3.2. Os dados dos alunos que concluíram todas as atividades foram analisados de duas formas: análise qualitativa e análise quantitativa. A análise qualitativa foi realizada com propósito exploratório, a fim de identificar aspectos subjetivos. A análise quantitativa foi feita por meio análises estatísticas a partir dos dados coletados da interação dos alunos.

\subsection{Análise Qualitativa}

Durante a aplicação do experimento, três pesquisadores-observadores analisaram o comportamento dos alunos em pontos específicos da atividade. Após a finalização de todas as etapas, escreveram um relato da experiência e das observações realizadas. Esses dados foram classificados e sumarizados pelos autores deste trabalho.

Durante a execução do ambiente E-Class, no grupo não gamificado, observou-se que alguns alunos tentaram consultar os colegas no intuito de obter a resposta para os exercícios. Diversas vezes houve a intervenção da instrutora a fim de evitar esse tipo de comportamento. Alguns alunos também tentaram observar a tela dos colegas para verificar em qual questão estavam respondendo. Além disso, foi observado o uso excessivo do botão "Dicas", quando perceberam que as dicas chegavam bem próximo da resposta do exercício. Houve muita dispersão da atenção e, em alguns casos, os alunos passaram nitidamente a vontade de apenas responder as questões e terminar a atividade rapidamente.

Por outro lado, o grupo que executou a atividade utilizando o E-Game demonstrou mais interesse, atenção e foco na atividade, em relação ao grupo de controle. Foi observado um demasiado interesse pelos pontos e ranking no E-Game, e os alunos ficavam eufóricos quando ultrapassavam os colegas no ranking. Em relação as dicas, pelo fato das regras do E-Game permitirem a abertura da terceira dica após a 'compra' com os pontos ganhos, os 
V Congresso Brasileiro de Informática na Educação (CBIE 2016)

Anais dos Workshops do V Congresso Brasileiro de Informática na Educação (CBIE 2016)

alunos demonstraram mais vontade em resolver as questões, ao invés de visualizar as dicas a todo momento. Além disso, a concentração dos alunos na atividade foi maior. Foi notório a competição entre os alunos, na qual eles não permitiam que os colegas visualizassem as respostas das suas questões. Durante essa atividade, os alunos demonstraram mais diversão, comemorando quando acertavam uma questão e quando ganhavam um novo badge.

\subsection{Análise Quantitativa}

Todos os dados coletados sobre a interação dos alunos com o ambiente educacional (EGame e E-Class) foram coletados e adequadamente tabulados. Em seguida, foi efetuada a análise da curva de distribuição geral dos dados, para averiguar se os dados obtidos estão condicionados a uma distribuição Normal. Adicionalmente, verificou-se, por inspeção visual, a distribuição dos dados por meio dos histogramas e gráficos do tipo box-plot de todas as métricas. Os resultados dos testes S-W detectaram que a distribuição dos dados era não normal (Sig. < 0,05). Por isso, optou-se por utilizar testes não paramétricos (MannWhithney) para análise e comparação dos dados.

Para responder a QP1 (resumidamente, a gamificação diminui o gaming the system?), verificou-se as variáveis: tempo de permanência nas questões, tempo para abrir a primeira dica, tempo lendo as dicas de 1 a 5 , quantidade de dicas solicitadas, tempo para escolher uma resposta e tempo de alteração das alternativas.

Primeiramente, o tempo de permanência nas questões foi analisado, identificando um tempo maior de permanência durante o uso do E-Game, como mostra a Figura 2. Essa variável significa que os alunos demoraram mais tempo realizando as questões no ambiente gamificado, em comparação ao ambiente não gamificado. Esses dados corroboram com as observações realizadas durante a análise qualitativa, onde foi observado durante o E-

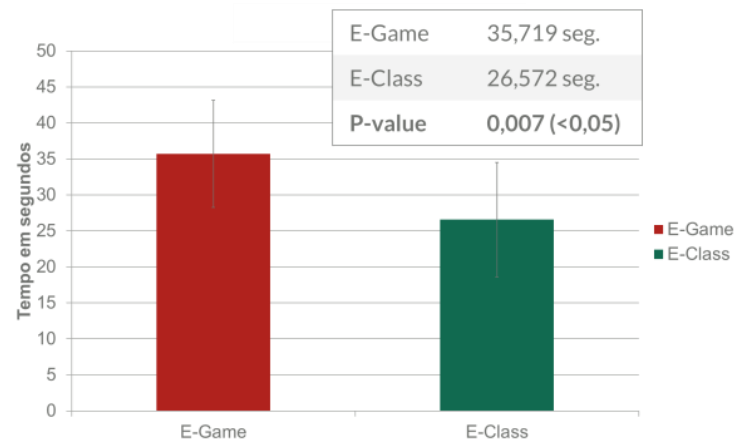

Figura 2. Tempo de permanência Class que os alunos queriam apenas terminar a atividade o mais rápido possível, enquanto que no E-Game os alunos levaram mais tempo para finalizar a atividade. Acredita-se que os alunos levaram mais tempo pelo fato dos elementos de jogos chamarem a atenção e motivarem os alunos a tentarem acertar as questões. Aplicando o teste estatístico de MannWhitney temos que $p=0,007$, ou seja, existe uma diferença significativa $(p<0,05)$ nos tempos de permanência em cada um dos grupos. Dessa forma, pode-se afirmar que os alunos passaram mais tempo interagindo com o E-Game em comparação com o E-Class.

Em relação aos gêneros masculino e feminino, os dados estatísticos obtidos na análise do E-Game e E-class mostra que o valor $p$ é menor que 0,05 (feminino $p=0,000$; masculino $p=0,035)$, ou seja, estatisticamente houve diferença entre os gêneros. No EGame, o gênero que se destacou por permanecer mais tempo nas questões foi o masculino, enquanto que no E-Class foi o gênero feminino. Além disso, a análise realizada entre o mesmo gênero em ambientes educacionais diferentes também obteve um resultado estatisticamente significativo. $\mathrm{O}$ gênero feminino permaneceu por mais tempo lendo as questões no E-Class, enquanto que o gênero masculino permaneceu mais tempo no EGame. Este fato oferece indícios de que o gênero feminino não demonstrou tanto interesse pelos elementos de gamificação, e pior ainda, a gamificação prejudicou a interação. 
A Figura 3 mostra o tempo em segundos que o aluno levou para ler a questão e fazer a solicitação da primeira dica. Esse tempo demonstra quanto o aluno gastou efetivamente lendo a questão, tentando resolvê-la, antes de solicitar as dicas. No EGame, os alunos demoraram mais tempo para fazer a solicitação da dica, em comparação com o grupo do E-Class. Entre os ambientes E-Game e E-Class, tanto o gênero masculino quanto o feminino demonstraram um tempo

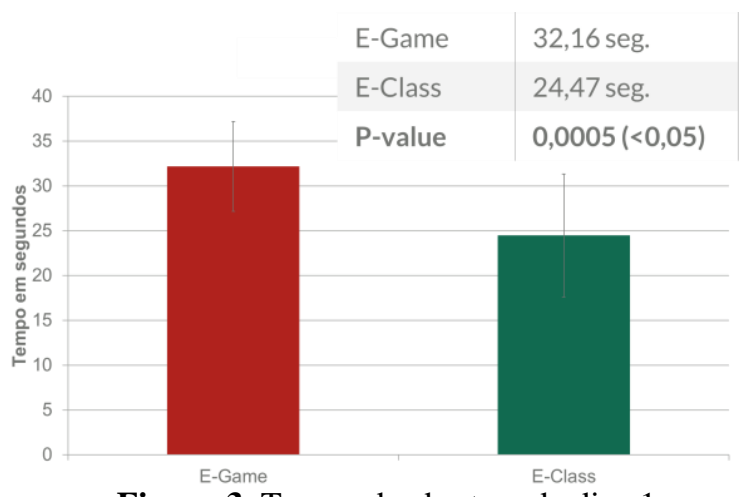

Figura 3. Tempo de abertura da dica 1 menor para solicitar a dica no E-Class, indicando que os alunos pensaram menos para responder uma questão no ambiente não gamificado. $\mathrm{O}$ resultado de Mann-Whitney demonstra significância na análise por gênero durante o uso do E-Game. $O$ gênero masculino obteve um resultado significativamente maior, ou seja, os meninos demoraram mais para fazer a solicitação das dicas do que as meninas, no ambiente gamificado. Entretanto, o gênero feminino obteve uma diferença significativa no E-Class, ou seja, elas demoraram mais para fazer a solicitação das dicas no ambiente não gamificado.

Uma outra variável analisada para medir o gaming the system é a quantidade de vezes e o tempo que o aluno utiliza para mudar as suas respostas. Em relação a essa variável, durante a atividade do E-Game, os alunos mudaram mais de alternativas do que na atividade do E-Class. Esse tipo de comportamento, analisado isoladamente, pode ser também considerado como indecisão. Foi detectado a troca nas alternativas de até seis vezes. Entretanto, essa variável deve ser observada junto ao tempo entre essas trocas. Aplicando o teste estatístico de Mann-Whitney nos dados obtidos da quantidade de troca e do tempo registrado, obtivemos que o tempo em relação as trocas das alternativas foi maior no E-Game em relação ao E-Class. Isso indica que houve um índice maior de "chutes" no E-Class, devido ao tempo curto de troca de alternativas.

Finalmente com relação aos dados do questionário motivacional IMI aplicado após a atividade, encontrou-se resultados significativos na categoria "Pressão/Tensão" $(p=0,044)$ para ambos os gêneros sugerindo que os alunos se sentiram mais focados (e pressionados) durante o uso do E-Game. Além disso, o gênero feminino obteve um resultado intrigante na categoria "Competência" $(p=0,028)$, pois elas se sentiram menos competente durante o uso do E-Game. De maneira geral, como apresenta a Figura 4, os alunos/sujeitos do gênero masculino apresentaram maior motivação ao utilizar o E-Game enquanto que as alunas/sujeitos do gênero feminino apresentaram maior motivação ao utilizar o E-Class.

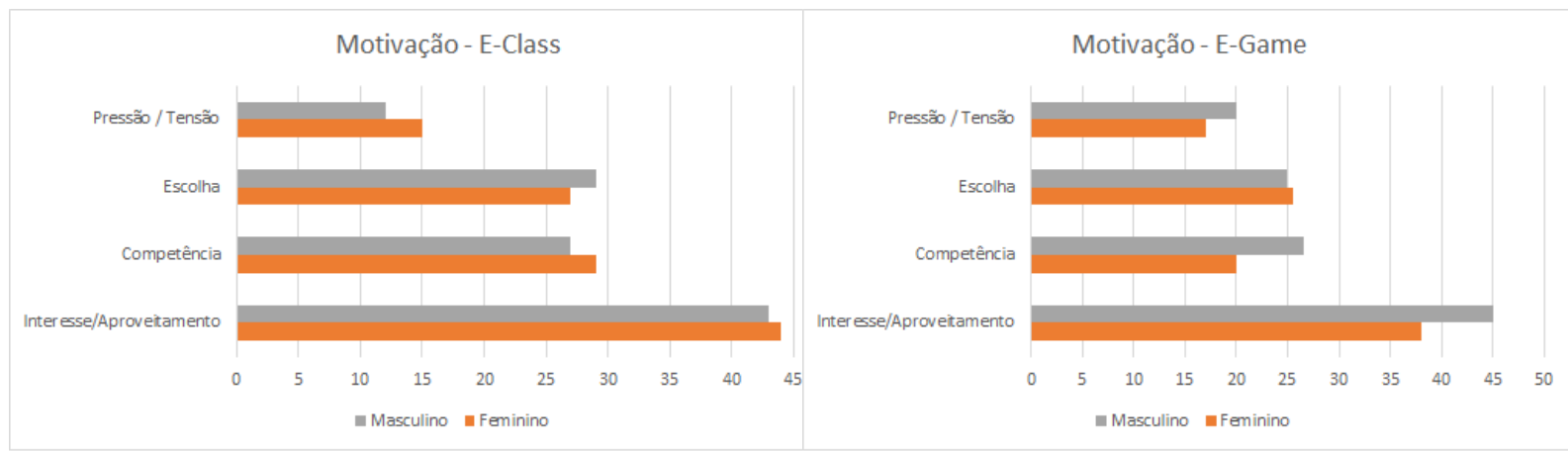

Figura 4. Resultados do questionário motivacional IMI com diferença entre gêneros 
V Congresso Brasileiro de Informática na Educação (CBIE 2016)

Anais dos Workshops do V Congresso Brasileiro de Informática na Educação (CBIE 2016)

\section{Conclusão}

A principal questão de pesquisa que norteou este trabalho foi QP1 (a gamificação diminui $o$ gaming the system?). Para responder esta pergunta estudou-se com especialistas em gamificação para identificar os elementos e mecânicas de jogos que alterariam positivamente o comportamento do aluno. Em seguida a gamificação foi implementada no ambiente educacional E-Game. Três experimentos foram realizados durante esta pesquisa (Pedro, 2016) e, devido a limitação de espaço, apenas um foi reportado.

Os resultados empíricos mostraram forte impacto na diminuição do gaming the system com o uso da gamificação. Além disso, identificou diferenças de comportamento e impacto motivacional entre os gêneros. Foi detectado que sujeitos do gênero masculino externalizaram menos trapaças durante o uso do E-Game, em comparação com sujeitos do gênero feminino. Além disso, sujeitos do gênero feminino se sentiram menos competentes e motivadas ao usar o ambiente gamificado. Uma das explicações para estes resultados é a forma como a gamificação é planejada atualmente, focando excessivamente nos elementos de competição (ranking, medalhas, pontos e etc). Esses resultados sugerem que do ponto de vista do usuário (user-centric approach) a gamificação precisa ser planejada levando em consideração também o gênero dos alunos.

Além dos resultados empíricos, temos também como contribuição deste trabalho (i) a publicação de artigos científicos (Pedro et al., 2015; Rocha et al., 2015; Lopes et al., 2014; Andrade et al., 2013); (ii) a tradução e validação do questionário motivacional IMI para o Português-Brasileiro; (iii) a parametrização do gaming the system permitindo que qualquer outro ambiente educacional possa estudar este tipo de comportamento; e (iv) o desenvolvimento do E-Game, uma ferramenta open source disponível como software livre em https://github.com/laiszp. Essa ferramenta poderá ser utilizada como base para realização de novas pesquisas empíricas sobre o impacto da gamificação na aprendizagem.

Salientamos que este é o primeiro trabalho que reporta a descoberta da relação entre elementos de gamificação, gaming the system e gênero, baseados em dados empíricos em um ambiente real de aprendizagem. Essa constatação é observada ao analisar os artigos científicos disponíveis em mapeamentos sistemáticos, como, Klock et al. (2014), Borges et al. (2015) e Dicheva et al. (2015), que exploraram centenas de artigos e reportam que apenas dois deles analisam dados dos gêneros masculino e feminino separadamente. Contudo, nenhum destes artigos explora o comportamento dos alunos. Acreditamos que este resultado pode servir de base para novas perspectivas e direções de pesquisa na área de gamificação da aprendizagem que poderão ser estudadas em pesquisas futuras.

\section{Referências}

Andrade, F. R. H.; Pedro, L. Z.; Lopes, A. M. Z.; Bittencourt, I. I.; Isotani, S. (2013) Desafio do uso de Gamificação em Sistemas Tutores Inteligentes baseados em Web Semântica. Anais do Congresso da Sociedade Brasileira de Computação, 1453-1462.

Baker, R. S. J. D.; Carvalho, A.; Raspat, J.; Aleven, V.; Corbett, A. T.; Koedinger, K. R. (2009) Educational software features that encourage and discourage gaming the system. Proceedings of the International Conference on Artificial Intelligence in Education, 475-482.

Baker, R.; Walonoski, J.; Heffernan, N.; Roll, I.; Corbett, A.; Koedinger, K. (2008) Why students engage in "gaming the system" behavior in interactive learning environments. Journal of Interactive Learning Research, 19(2), 185-224.

Borges, S. S.; Durelli, V.; Reis, H. M.; Isotani, S. (2015) A systematic mapping on gamification applied to education. Proceedings of the ACM Symposium on Applied Computing, 216-222. 
V Congresso Brasileiro de Informática na Educação (CBIE 2016)

Anais dos Workshops do V Congresso Brasileiro de Informática na Educação (CBIE 2016)

Cetintas, S.; Si, L.; Xin, Y. P.; Hord, C. (2010) Automatic detection of off-task behaviors in intelligent tutoring systems with machine learning techniques. IEEE Transactions on Learning Technologies, 3(3), 228-236.

Deci, E. L.; Ryan, R. M. (2011) Levels of analysis, regnant causes of behavior and well-being: The role of psychological needs. Psychological Inquiry, v. 22, p. 17-22.

Dicheva, D.; Dichev C.; Agre G.; Angelova G. (2015) Gamification in Education: A Systematic Mapping Study. Educational Technology \& Society, 18 (3), 75-88.

Doddannara, L. S.; Gowda, S. M.; Baker, R.; Gowda, S. M.; Carvalho, A. (2013) Exploring the relationships between design, students' affective states, and disengaged behaviors within an ITS. Proceedings of the International Conference on Artificial Intelligence in Education, 31-40.

Giannakos, M. N.; Chorianopoulos, K.; Jaccheri, L. (2012) Math is not only for Science Geeks: Design and Assessment of a Storytelling Serious Video Game. Proceedings of the IEEE International Conference on Advanced Learning Technologies, 418-419.

Hess, T.; Gunter, G. (2013) Serious game-based and nongame-based online courses: Learning experiences and outcomes. British Journal of Educational Technology, 44(3), 372-385.

Isotani, S.; Mizoguchi, R.; Bittencourt, I.; Costa, E. (2009) Estado da arte em web semântica e web 2.0: Potencialidades e tendências da nova geração de ambientes de ensino na internet. Revista Brasileira de Informática na Educação, 17(1), 30-42.

Kapp, K. M. (2012) The Gamification of Learning and Instruction: Game-based Methods and Strategies for Training and Education. John Wiley \& Sons.

Klock, A. C. T.; Gasparini, I.; Kemczinski, A.; Hounsell, M.; Isotani, S. (2015) One man's trash is another man's treasure: um mapeamento sistemático sobre as características individuais na gamificação de ambientes virtuais de aprendizagem. Anais do Simpósio Brasileiro de Informática na Educação, 539-548.

Lopes, A. M. Z.; Pedro, L. Z.; Isotani, S. (2014) Qualidade de Softwares Educacionais Baseados na Web. RENOTE - Revista Novas Tecnologias na Educação, 12(1), 1-10.

Mayer, I.; Warmelink, H.; Bekebrede, G. (2013) Learning in a game-based virtual environment: A comparative evaluation in higher education. European Journal of Engineering Education, 38(1), $85-106$.

Muldner, K.; Burleson, W.; Van de Sande, B.; VanLehn, K. (2011) An analysis of students' gaming behaviors in an intelligent tutoring system: Predictors and impacts. User Modeling and User-Adapted Interaction, 21(1-2), 99-135.

Pedro, L. Z.; Lopes, A. M. Z.; Prates, B. G.; Vassileva, J.; Isotani, S. (2015) Does Gamification Work for Boys and Girls? An Exploratory Study with a Virtual Learning Environment. Proceedings of the ACM Symposium on Applied Computing, 214-219.

Pedro, L. Z. (2016) Uso de gamificação em ambientes virtuais de aprendizagem para reduzir o problema da externalização de comportamentos indesejáveis. Dissertação de Mestrado em Ciência da Computação. Universidade de São Paulo.

Rocha, R. V. C.; Lopes, A. M. Z.; Pedro, L. Z.; Bittencourt, I.; Isotani, S. (2015) Metodologia de Desenvolvimento de Jogos Sérios: Especificação de Ferramentas de Apoio Open Source. Anais do Simpósio Brasileiro de Informática na Educação, 489-498.

Simpson, O. (2013). Supporting students in online open and distance learning. Routledge.

VanLehn, K. A.; Burleson, W. S.; Girard, S.; Chavez-Echeagaray, M. E.; Gonzalez-Sanchez, J.; Hidalgo-Pontet, Y.; Zhang, L. (2014) The Affective Meta-Tutoring Project: Lessons Learned. Proceedings of the International Conference on Intelligent Tutoring Systems, 84-93. 